\title{
Live vaccine consisting of attenuated Salmonella secreting and delivering Brucella ribosomal protein L7/L12 induces humoral and cellular immune responses and protects mice against virulent Brucella abortus 544 challenge
}

Amal Senevirathne, Chamith Hewawaduge and John Hwa Lee* (]

\begin{abstract}
The present study employs the Brucella abortus L7/L12 antigen in a Salmonella secretion platform and investigates its ability to induce protective immune responses against wild type challenge in BALB/c mice. The highly conserved L7/ L12 open reading frame was PCR amplified from B. abortus and cloned into a prokaryotic expression vector, pJHL65, directly under the beta-lactamase secretory signal. The plasmid constructs pJHL65::L7/L12 was then transformed into an attenuated Salmonella Typhimurium strain, JOL1800 ( $\Delta / o n, \Delta c p x R$, $\triangle a s d$, and $\Delta r f a L)$, and protein secretion was verified by Western blot. Three mice groups were inoculated with either phosphate-buffered saline (PBS), vector-only control, or the vaccine strain secreting L7/L12 antigen. Assessment of humoral and cell-mediated immune responses revealed successful elicitation of Brucella antigen-specific Th1 and Th2 immune responses that were significantly higher than PBS and vector control groups. The immune responses were confirmed by splenocyte proliferation assay, flow cytometry analysis for CD4+ and CD8+ markers, and RT-PCR based cytokine profiling upon restimulation with $L 7 / L 12$ purified antigen. Results indicate that immunization with Salmonella secreting L7/L12 antigen demonstrated significant enhancement of cell-mediated immune (CMI) responses in immunized mice. The overall effectiveness of the immunization was evaluated by challenging with virulent B. abortus that revealed significant reduction in Brucella colonization in spleen and liver tissues in Salmonella L7/L12 immunized mice. Delivery of Brucella protective antigen L7/L12 using the Salmonella secretion system can effectively accomplish immunogenic advantages of both Salmonella and L7/L12 to derive robust CMI responses and induce humoral immunity to protect against Brucella infection in the mouse model.
\end{abstract}

${ }^{*}$ Correspondence: johnhlee@jbnu.ac.kr

College of Veterinary Medicine, Chonbuk National University, Iksan

Campus, Iksan 54596, Republic of Korea

\begin{abstract}
Introduction
Brucella abortus is a zoonotic bacterial pathogen within the genus Brucella. It induces abortions, placental retention, and infertility in ruminants and undulant fever in humans with occasional chronic infections. As an intracellular pathogen, it proliferates in both phagocytic [1] and non-phagocytic cells [2] in the infected host.
\end{abstract}

c) The Author(s) 2020. This article is licensed under a Creative Commons Attribution 4.0 International License, which permits use, sharing, adaptation, distribution and reproduction in any medium or format, as long as you give appropriate credit to the original author(s) and the source, provide a link to the Creative Commons licence, and indicate if changes were made. The images or other third party material in this article are included in the article's Creative Commons licence, unless indicated otherwise in a credit line to the material. If material is not included in the article's Creative Commons licence and your intended use is not permitted by statutory regulation or exceeds the permitted use, you will need to obtain permission directly from the copyright holder. To view a copy of this licence, visit http://creativeco mmons.org/licenses/by/4.0/. The Creative Commons Public Domain Dedication waiver (http://creativecommons.org/publicdomain/ zero/1.0/) applies to the data made available in this article, unless otherwise stated in a credit line to the data. 
Host resistance to $B$. abortus mainly depends on interferon-gamma (IFN- $\gamma$ ) involved cell-mediated immune responses [3]. Up regulation of IFN- $\gamma$ cytokine is essential to induce the anti-Brucella activity of macrophages that helps to clear Brucella from the infected host [4].

Among antigens that efficiently induce anti-Brucella cell-mediated immune (CMI) responses, Brucella ribosomal protein L7/L12 has been recognized as a plausible candidate in recent research studies. These experiments have shown that L7/L12 can efficiently induce protective immune responses in the form of subunit or DNA vaccines [5, 6]. Apart from CMI responses, antibodymediated humoral responses also play a vital role during $B$. abortus infection. Elicitation of mucosal immune responses is effective during the early phase of infection on mucosal surfaces, where the complement killing system can reduce the proportion of Brucella entering the intracellular environment [7]. Therefore, an effective prevention strategy for $B$. abortus should ideally harness both CMI responses and humoral immune responses in the immunized host [8]. Utilizing an attenuated systemic pathogen such as Salmonella for the delivery of protective antigens can capitalize on both CMI and humoral responses considering their mechanism in host immune stimulation. Based on the above fact, here we utilize Salmonella to deliver Brucella antigen (L7/L12). As an intracellular pathogen, Salmonella can invade into host macrophages and lymphoid organs such as the spleen and liver [9]. Thus, Salmonella can effectively deliver heterologous antigens directly into macrophages, other cells, and tissues where antigen-presenting cells can process and recognize Brucella antigens for adaptive immune responses and fight against infections. Further, Salmonella can overcome mucosal barriers and actively penetrate the host tissue environment that is essential to maximize antigen presentation [10]. In addition, sufficient attenuation can also be incorporated into the Salmonella genome with relative ease due to its genome that has been thoroughly investigated with most virulence-related genes being identified.

Despite scientific advancement in DNA vaccine technology during recent years, plasmid delivery in in vivo conditions remains a significant challenge. Intracellular delivery, entry into the cell nucleus, and expression of the intended protein is a relatively inefficient process. To overcome such inefficacy in DNA vaccines, high quantities of plasmid DNA usually have to be utilized with multiple booster applications [11]. Such inconveniences may be avoided by using an efficient Salmonella mediated secretion system that does not require induction or multiple immunizations. In our previous study, an attenuated Salmonella Typhimurium strain, JOL1800, was constructed with specific deletions in the lon, $c p \times R$, and $r f a L$ genes. The resulting strain has impaired intracellular survival due to loss of lon and $c p x R$ virulence-related genes [12] and has a truncated lipopolysaccharide structure due to a $r f a L$ gene mutation. The resulting JOL1800 DIVA (Differentiating infected from vaccinated animals) is ideal for vaccine studies. Besides, this strain is aspartate dehydrogenase auxotrophic $(\Delta a s d)$ and can stably harbor plasmid constructs complemented with asd gene [13].

The aim of the present study is to design a safe Brucella vaccine that is applicable in both human and animal models for efficient elicitation of humoral and cell-mediated immune responses utilizing a Salmonella secretion and delivery system. The present Salmonella strain, JOL1800, is an asd auxotrophic mutant that can stably carry an asd complemented plasmid pJHL65, which contains fusion of $B$. abortus ribosomal protein L7/L12 with the beta-lactamase (bla) secretory signal sequence. Attenuated Salmonella can secrete heterologous Brucella antigens into the host by crossing the periplasmic barrier acting as an intracellular pathogen. Due to impaired virulence, attenuated Salmonella does not cause systemic infection and only provides transient immune stimulation in the host. We have observed robust elicitation of both humoral and cellular immune responses in immunized mice with significant protection against virulent challenges. We have observed Salmonella alone carrying the empty plasmid vector provide considerable protection against the virulent Brucella challenge that may be attributed to Salmonella induced immune stimulation. The results indicate that Salmonella is an excellent antigen delivery system for specific induction of anti-Brucella immunity that takes advantage of both Salmonella and L7/L12 immunogenic capabilities. This provides an easy and effective strategy for mass production of an anti-Brucella vaccine that is applicable to both humans and animals. In the present study, we demonstrated that a Salmonella system could be effectively utilized for delivery of Brucella ribosomal protein L7/L12. This elicits robust anti-Brucella protective immunity by achieving the immunogenic advantages of Salmonella and L7/L12 as a protective antigen to be used in the battle against Brucella infection.

\section{Materials and methods}

\section{Bacterial strains and plasmids}

The bacterial strains, plasmids, and primers used in this experiment are listed in Table 1. Attenuated Salmonella and Escherichia coli strains were routinely grown at $37{ }^{\circ} \mathrm{C}$ in Luria-Bertani (LB) broth or agar with or without appropriate antibiotics unless otherwise indicated. B. abortus 544 strain was grown in Brucella medium (BD, Sparks, USA) at $37{ }^{\circ} \mathrm{C}$ in a $5 \% \mathrm{CO}_{2}$ atmosphere 
Table 1 Bacterial strains, plasmids, and primers

\begin{tabular}{|c|c|c|}
\hline Strain/Plasmid & Description & Reference \\
\hline \multicolumn{3}{|l|}{ S. Typhimurium } \\
\hline JOL1800 & $\Delta o n, \triangle c p x R, \triangle a s d$ and $\triangle w p a B$ mutant of $S$. Typhimurium & Lab stock \\
\hline $\mathrm{JOL} 2273$ & JOL1800 containing pJHL65:: L7/L12 and expressing I7/L12 & Lab stock \\
\hline JOL2080 & JOL1800 carrying the empty vector & Lab stock \\
\hline \multicolumn{3}{|l|}{ E. coli } \\
\hline BL21(DE3)pLysS & $\mathrm{F}^{-}, \mathrm{ompT}, \mathrm{hsdS_{B }}\left(\mathrm{r}_{\mathrm{B}}^{-}, \mathrm{m}_{\mathrm{B}}^{-}\right), \mathrm{dcm}, \mathrm{gal}, \lambda(\mathrm{DE} 3), \mathrm{pLys} \mathrm{S}, \mathrm{Cm}^{r}$ & Progma, USA \\
\hline JOL1921 & BL21(DE3)pLysS harboring pET28a + :: L7/L12 & Lab stock \\
\hline \multicolumn{3}{|l|}{ Plasmid } \\
\hline $\mathrm{pET} 28 \mathrm{a}(+)$ & IPTG-inducible expression vector; Kanamycin resistant & Novagen, USA \\
\hline pET28a +:: L7/L12 & pET28a + derivative containing L7/L12 & Lab stock \\
\hline pJHL65 & $\begin{array}{l}\text { asd }^{+} \text {vector, pBR ori, } \beta \text {-lactamase signal sequence-based periplasmic secretion plas- } \\
\text { mid, } 6 x \text { His, high copy number }\end{array}$ & Lab stock \\
\hline pJHL65:: L7/L12 & pJHL65 harboring L7/L12 & Lab stock \\
\hline \multicolumn{3}{|l|}{ Primer } \\
\hline L7/L12 EcoRI F & 5'-AGAGGAATTCATGGCTGATCTCGCAAAGAT-3' & This study \\
\hline L7/L12 HindIII R & 5'-AGAGAAGCTTTTACTTGAGTTCAACCTTGG-3' & This study \\
\hline
\end{tabular}

without antibiotics. Appropriate biosafety measures were enforced while handling B. abortus, a biosafety level 3 microorganism.

\section{Construction of attenuated Salmonella mutant delivering Brucella antigen}

The construction of the asd complemented pJHL65 plasmid that constitutively expresses heterologous antigens was described in a previous report [7]. Briefly, Brucella ribosomal protein L7/L12 gene was PCR amplified from the $B$. abortus genome and cloned in-frame immediately downstream to the beta-lactamase (bla) secretory signal. Thereby, proteins can be externalized using a Type II secretory system. The novel plasmid construct was denoted as pJHL65:: L7/L12. The recombinant plasmid was then transformed into an asd mutant auxotrophic Salmonella mutant strain JOL1800 (Alon, $\triangle c p x R, \Delta a s d$, and $\Delta r f a L)$. The resultant strain was designated as JOL2273. The presence of the respective gene in the attenuated Salmonella strain was confirmed by PCR. Secretion of Brucella ribosomal protein L7/L12 by the attenuated Salmonella strain was confirmed by Western blot analysis utilizing HRP labeled anti-his tag antibody. To generate purified protein, the L7/L12 coding sequence was cloned into a pET28a+ vector (Novagen, Madison, WI, USA). The recombinant plasmid was then transformed into E. coli BL21 DE strain (Novagen) for overexpression and purification. The expressed protein was purified using Ni_NTA (Qiagen, Valencia, CA, USA) affinity column chromatography (Poly-prep, BioRad, Hercules, CA, USA) according to the manufacturer's instructions. The concentration of purified protein was then quantified by Bradford assay and stored at $-20{ }^{\circ} \mathrm{C}$ for further use.

\section{Immunization and challenge}

All animal experiments were approved by the Chonbuk National University Animal Ethics Committee (CBNU2018-00264) in accordance with the guidelines of the Korean Council on Animal Care and Korean Animal Protection Law, 2007; Article 13. All mice used in the study were housed and maintained humanely with the ad libitum supply of antibiotic-free food and water. Five-week old specific pathogen-free female BALB/c mice $(n=10)$ were randomly divided into three groups: PBS control group, vector-only group, or L7/L12 treatment group. Each mouse group was intra-muscularly immunized with phosphate-buffered saline (PBS), Salmonella JOL1800 bearing only the vector, or treatment strain harboring pJHL65::L7/L12 at $1 \times 10^{7} \mathrm{CFU} /$ mice in $100 \mu \mathrm{L}$ of PBS. On the 14th day post-immunization, blood samples for serum preparation $[14,15]$ and vaginal washes were collected [7] for IgG and sIgA and subtype IgG and IgG2a antibody assessment $(n=10)$. In addition, on the 14th day, five mice from each group were sacrificed for splenocyte collection and used for splenocyte proliferation assay, flow cytometry analysis, and RT-PCR assays. Twenty-one days post-immunization, the remaining mice $(n=5)$ from each group were intraperitoneally challenged with virulent $B$. abortus 544 strain at $2 \times 10^{5} \mathrm{CFU} /$ mouse. After 14 days of challenge, all mice were euthanized. The total Brucella load in whole spleen and liver 
organs were enumerated by counting colony-forming units using macerated tissue samples as described before [7].

\section{Antigen-specific $\lg G$ and secretory $\lg A$ responses} Indirect ELISA (enzyme-linked immunosorbent assay) was used to assess L7/L12 specific IgG and secretory IgA (sIgA) responses in sera and vaginal wash samples, respectively, according to a previous report [16] with minor modifications. Briefly, purified L7/L12 protein (300 ng/well) was coated on the ELISA plate overnight at $4{ }^{\circ} \mathrm{C}$ in a coating buffer. Plates were washed and blocked with $5 \%$ skim milk. Serum samples (1:50) and vaginal washes (without dilution) were incubated for $1 \mathrm{~h}$ at $37{ }^{\circ} \mathrm{C}$. Unbound antigen was washed, and plates were incubated with anti-his tag HRP (1:3000). Color development was achieved by adding o-phenylenediamine dihydrochloride (OPD) substrate and was measured at $492 \mathrm{~nm}$ wavelength. Further, serum collected at 3rd week post-immunization was assessed in ELISA against L7/ L12 purified protein, Brucella whole cell lysate and Salmonella whole cell lysate following the same procedure.

\section{Antigen-specific splenocyte proliferation assay}

Antigen-specific cell-mediated immune responses (CMI) were evaluated in a splenocyte proliferation assay. Splenocytes from mice $(n=5)$ in each PBS control, vector alone, and Salmonella L7/L12 groups were harvested at 2 and 3 weeks of post-immunization. Cell number was adjusted to $1 \times 10^{5}$ cells/well in 96-well plates and seeded in replicates using RPMI medium supplemented with $10 \%$ FBS. Next, cells were stimulated with specific purified L7/L12 protein $10 \mu \mathrm{g} / \mathrm{mL}$ or RPMI media alone. Three days later, cell proliferative response was evaluated by MTT [3-(4,5Dimethylthiazol-2-yl)-2,5-diphenyltetrazolium bromide] based assay as described in an earlier report [17]. The stimulation index was calculated compared to the responses received in PBS control group. To further confirm the CMI response, the same experiment was repeated at 3rd week post-immunization and stimulation either with purified Brucella L7/L12 protein or $B$. abortus whole cell lysate $\left(10^{7} \mathrm{CFU} / \mathrm{mL}\right)$. Splenocytes collected at 3 weeks post-immunization were tested against L7/L12 purified protein and Brucella whole cell lysate for comparison.

\section{Cytokine responses}

Splenocytes from immunized mice were aseptically harvested using five mice per group. Splenocytes $\left(1 \times 10^{6}\right.$ cells $\left./ \mathrm{mL}\right)$ were stimulated with $200 \mathrm{ng}$ of the respective purified L7/L12 antigen protein for $24 \mathrm{~h}$. Total RNA was isolated by RNeasy Mini Kit (Qiagen, Hilden, Germany) according to the manufacturer's instructions.
Complementary DNA was prepared from $1 \mu \mathrm{g}$ of RNA using ReverTra Ace qPCR RT Master Mix (TOYOBO, Osaka, Japan) according to the manufacturer's instructions and stored at $-20{ }^{\circ} \mathrm{C}$ until use. Real-time PCR assay (qRT-PCR) for gene expression studies was performed with ABI applied biosystems using Power SYBR Green PCR Master Mix (\#4367659, Applied Biosystems, USA) as previously described [18]. The relative amounts of IFN- $\gamma$, IL-4, and IL-17 were determined by $2^{-\Delta \Delta C T}$ method [19].

\section{Flow cytometry}

Flow cytometric analysis of CD4+ and CD8+ T cell populations were carried out on mice splenocytes $(n=5)$ harvested on the 14th-day post-primary immunization, as previously described [7]. Briefly, $2 \times 10^{5}$ viable cells were stimulated with Brucella whole cell lysate antigen $(10 \mu \mathrm{g} / \mathrm{mL})$ or RPMI media alone for $24 \mathrm{~h}$ at $37^{\circ} \mathrm{C}$ in $5 \%$ $\mathrm{CO}_{2}$ atmosphere. Cells were then harvested and stained with PE-labeled anti-CD3e, PerCPVio700-labeled antiCD4, and FITC-labeled anti-CD8a monoclonal antibodies, as previously described [20]. The CD3+ T cell population was gated to analyze CD3+ CD4+ and CD3+ $\mathrm{CD} 8+$ subpopulations in both vaccinated and control mice groups using a MACSQuant ${ }^{\circledR}$ analyzer (Miltenyi Biotec, Bergisch Gladbach, Germany).

\section{Statistical analysis}

All data were analyzed using the GraphPad Prism 6.00 program (San Diego, CA, USA). One way analysis of variance (ANOVA) with Tukey's multiple comparison test was conducted to determine statistical differences among vaccinated and control groups. $P$ values $<0.05$ were considered statistically significant.

\section{Results}

Confirmation of Salmonella secreted Brucella antigen

The gene of $B$. abortus ribosomal protein L7/L12 was amplified by PCR using B. abortus 544 strain and cloned into the pJHL65-asd complemented plasmid (Additional file 1A). The insertion of the L7/L12 gene cassette $(0.374 \mathrm{~kb})$ was confirmed by PCR amplification and double digestion of host plasmid with EcoRI and HindIII restriction enzymes. The presence of the plasmid in Salmonella transformants was confirmed by colony PCR method. The pJHL65 plasmid provides bla secretion signal upstream to the L7/L12 gene cassette. The efficacy of protein secretion was confirmed by Western blot analysis of the culture supernatant, which yielded the target 
protein antigen with approximately $12.5 \mathrm{kDa}$ (Additional file 1B).

\section{Brucella antigen-specific systemic $\lg G$ and secretory $\lg A$ antibody response}

The degree of host response towards foreign antigen L7/ L12 was assessed by measuring the antigen-specific antibody response in blood serum and mucosal secretions. Efficient elicitation of both IgG and sIgA (Figure 1A) responses indicates the effectiveness of Salmonella delivered antigen presentation. Antibody concentrations on the 14th-day post-immunization demonstrate a significant high IgG and SIgA response in the L7/L12 treated mice group compared to the PBS and vector control groups. The specific elicitation of IgG1 and IgG2a antibody subtypes indicates engagement of both Th1 and Th2 type immune response upon Salmonella immunization. However, concerning IgA response, and IgG1 and IgG2a antibody subtype response, a significantly high antibody elicitation is evident due to Salmonella vector alone. This suggests that approximately $50 \%$ of IgA response, $55 \%$ IgG1, and 45\% IgG2a response elicited by the Salmonella secreting L7/L12 is attributed to Salmonella itself. Even though the vector alone elicits L7/L12 specific humoral responses, it is clear after statistical comparison that the Salmonella L7/L12 strain still generates significantly higher humoral response even after the subtraction of the vector alone responses. Further, to dissect
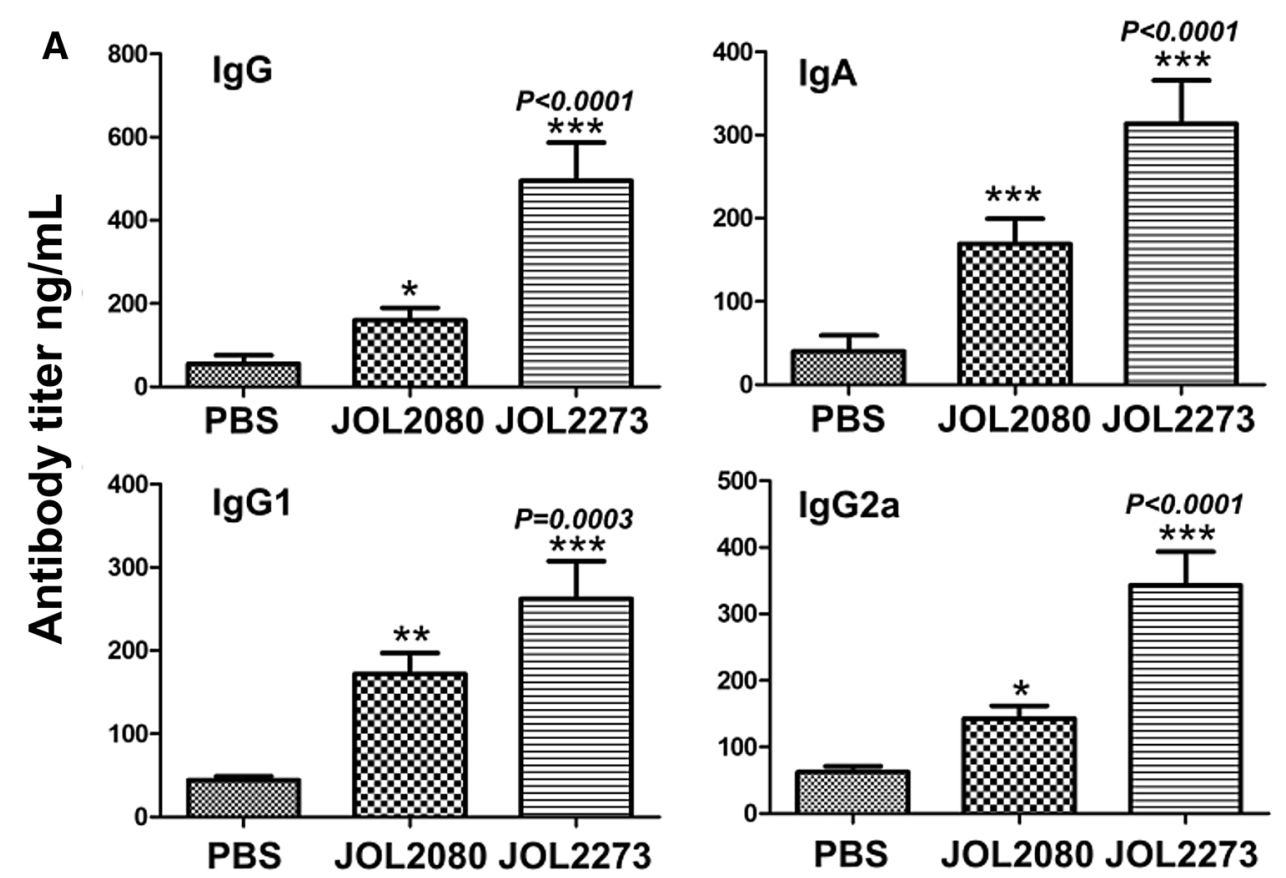

B
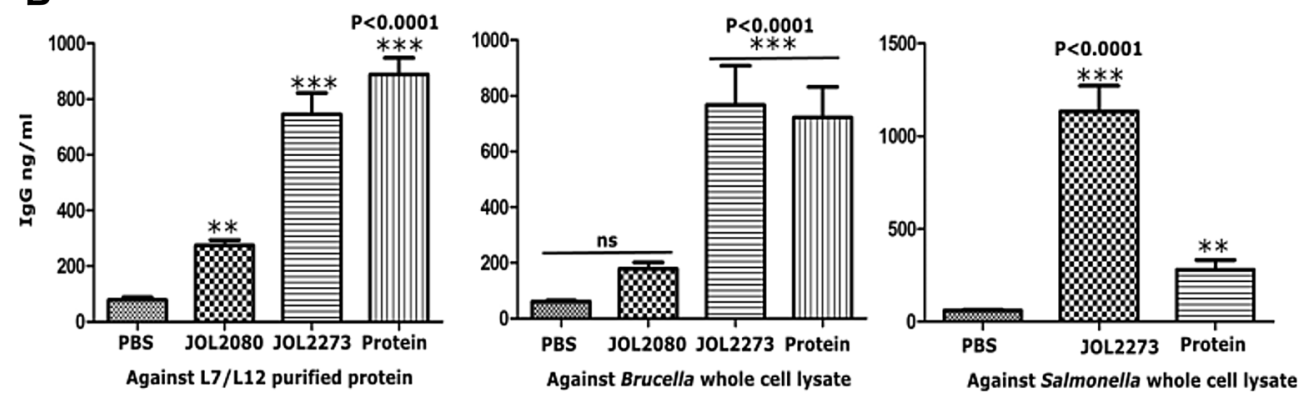

Figure 1 Humoral immune responses. Five-week old female BALB/c mice were immunized with PBS, vector-only strain, and Salmonella secreting Brucella L7/L12. Humoral responses in serum and vaginal washes were measured on 14th day post-immunization. A Serum IgG response, IgA response in vaginal washes, and serum IgG1 and lgG2a were demonstrated. *Indicates significant difference compared to PBS control $(P<0.05)$. Probability values were depicted. B The lgG responses at 21st day post-immunization were measured against purified L7/L12 antigen, Brucella whole cell lysate and Salmonella whole cell lysate were conducted. ${ }^{* *}$ Indicates the significant difference against the PBS control $(P<0.05)$. 
whether L7/L12 specific antibodies can cross react with Salmonella, a repeat experiment was carried out by inoculating mouse with purified protein along with PBS, Salmonella L7/L12 and Salmonella alone controls. Serum was collected after 3 weeks post-immunization tested against L7/L12 purified protein, Brucella whole cell lysate and Salmonella whole cell lysate (Figure 1B). Herein, the Salmonella vector alone response showed a reduced signal against Brucella whole cell lysate probably due to competing antigens and impurities present in the lysate. Further, this result confirms that Brucella abortus L7/L12 demonstrates a positive reaction against Salmonella whole cell lysate suggesting the fact that there can be a cross reaction between L7/L12 antibodies derived against Salmonella native L7/L12 antigen and Salmonella secreted Brucella L7/L12 antigen. It also can be hypothesized, that the antibodies developed against Brucella L7/L12 can result in a cross reaction with native Salmonella L7/L12 antigen due to higher amino acid sequence identity and homology present between the two species (Additional file 4).

\section{Cell-mediated immune responses}

The splenocyte proliferation assay is an indicator of CMI responses. The Brucella L7/L12 antigen is known to induce CMI responses. Thus, the retained biological activity of Salmonella secreted Brucella L7/L12 can be assessed by a MTT based splenocyte proliferation test. On the 14th day post-immunization, the L7/L12 treated group had significantly high cell proliferation response after re-stimulation of cells with purified L7/L12 protein compared to the PBS and vector control groups (Figure $2 \mathrm{~A}$ ). When the splenocytes were collected at 3 weeks post-immunization and stimulated by L7/L12 purified protein or Brucella whole cell lysate, proliferation test results demonstrated a higher specificity towards L7/ L12 antigen and reactivity against both purified L7/L12 protein and Brucella whole cell lysate at 3rd week postimmunization. This result indicates that Salmonella secreted L7/L12 elicits cell mediated immune responses that react when encounter immunizing antigen. The engagement of both Th1 and Th2 immune responses could be assessed by analyzing cytokine profiles. Herein, representative cytokines IFN- $\gamma$ and IL-4 expression levels show significant increase compared to the PBS control. Here too, vector alone responses also resulted in a significant difference from the PBS group. These results suggest that immunization with Salmonella secreting L7/L12 elicits a skewed response towards Th1 immune response over Th2 immune response, signifying an essential IFN$\gamma$ mediated cellular responses (Figure 2C). Furthermore, flow cytometry analysis of splenic T-cell populations after 14 days of immunization showed a significant increase in both $\mathrm{CD} 4+$ and $\mathrm{CD} 8+$ expressing cell populations. It suggests that Salmonella secreting Brucella L7/L12 antigen activates and increases antigen-specific T-cell responses in the host. The present results also argue that Salmonella alone elicits a considerable immune response against L7/L12 antigen stimulation.

\section{Protection efficacy against virulent Brucella challenge}

To investigate the protective efficacy of Salmonella delivered L7/L12 antigen against virulent $B$. abortus challenge, all immunized and control mice were challenged with $B$. abortus 544 strain. Two weeks after the challenge, Brucella bacterial burden was quantified by plate method with serially diluted tissue samples (Figure 3 ). Our results indicate approximately $2 \log$ reduction in bacterial numbers in both spleen and liver samples of the L7/L12 treatment group compared to the PBS and vector-only control groups (Figures $3 \mathrm{~A}$ and B). Protective index was calculated by using the statistical formula $(y=\log (x / \log$ $\mathrm{x}), \mathrm{PI}=\mathrm{y}_{\mathrm{pbs}}-\mathrm{y}_{\text {test }}$ ) (Figures $3 \mathrm{C}$ and $\mathrm{D}$ ). In the protection index too, Salmonella vector control resulted in a significantly high level of protection compared to the PBS control.

\section{Discussion}

The present study uses the highly immunogenic Brucella ribosomal protein L7/L12 in a Salmonella secretion platform as a live attenuated vaccine for brucellosis. The protective efficacy against virulent Brucella challenge was assessed in a BALB/c mice model. The combined elicitation of humoral and cell-mediated immune response clearly suggests that the combination of L7/L12 antigen with a Salmonella delivery system can increase the immune stimulation effect of both entities, Salmonella and L7/L12. The selected antigen L7/L12 consists of several dominant antigenic epitopes that are surface exposed (Additional file 2). Thus, efficient antigen processing and presentation are expected. Another significant advantage of L7/L12 ribosomal protein is its high sequence conservation, with more than $99 \%$ of its amino acid sequence identified in members of the Brucella genus (Additional file 3). Hence, cross-protection is also anticipated. In the present vaccine design, L7/L12 is incorporated into the Salmonella type II secretion system [21] via fusion with bla signal sequence and under the constitutive Ptrc expression promoter. This results in continuous antigen secretion into the surroundings (Additional file 1B). This may enhance the chances of antigen presentation by Salmonella secreting L7/L12 antigen as the Salmonella can penetrate the host tissues including the lymphoid organs such as spleen and liver $[22,23]$.

Despite recent research advancements in DNA vaccine technology, the efficacy of DNA delivery remains 

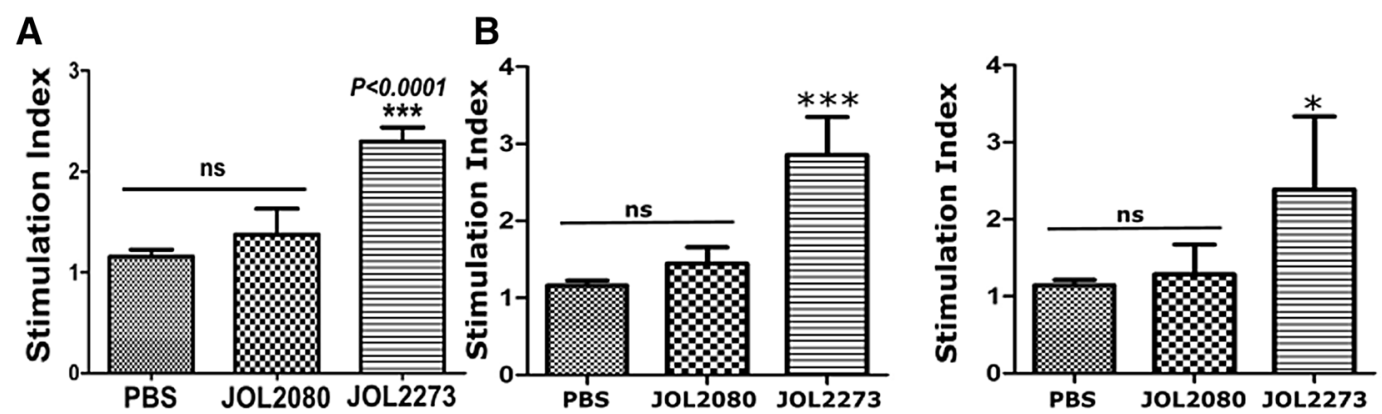

Stimulated with L7/L12 Stimulated with whole Brucella lysate
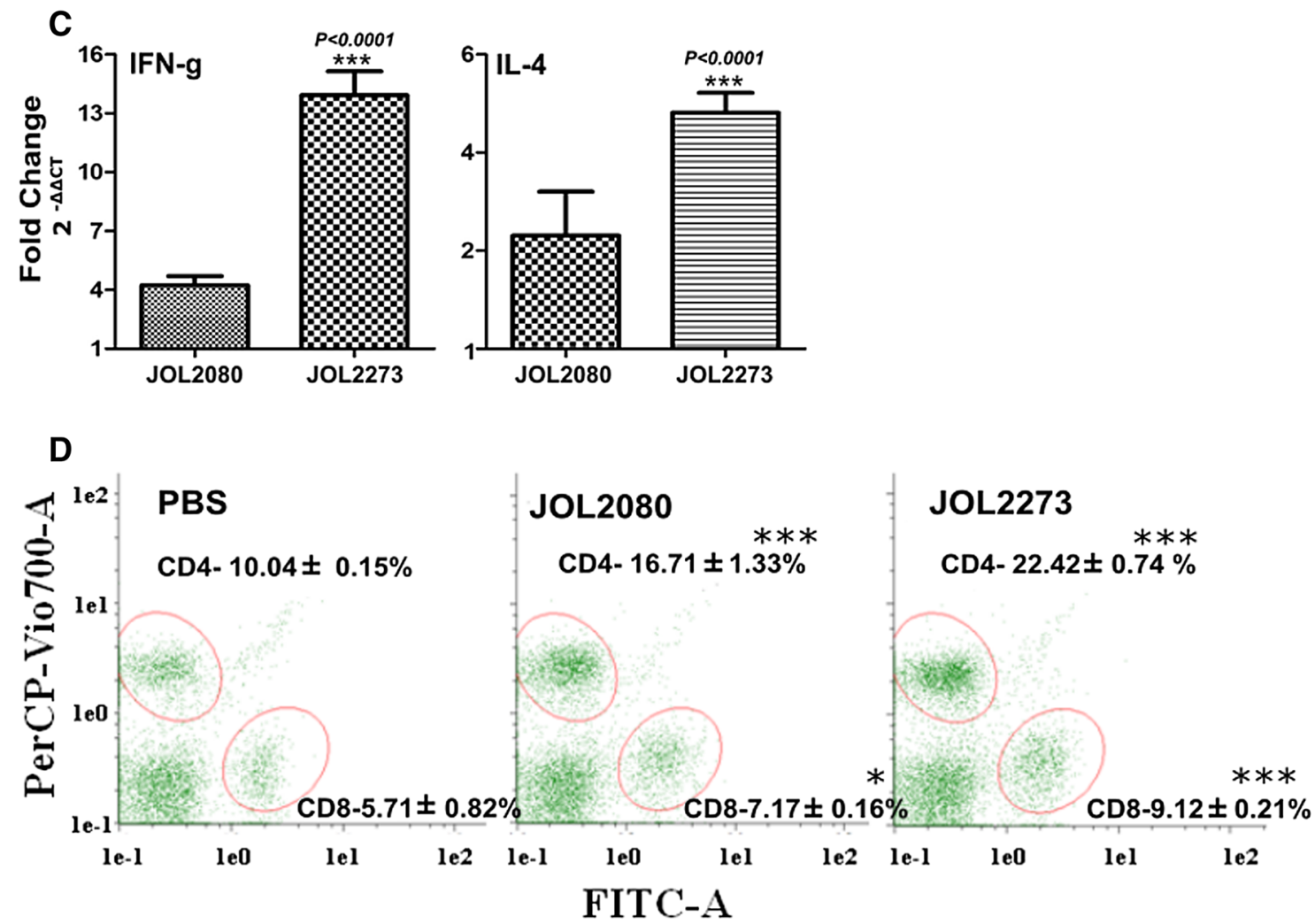

Figure 2 Cell mediated immune responses. A Stimulation index. Mice were immunized with PBS, vector-only strain, and Salmonella secreting Brucella L7/L12. Two weeks post-immunization, 5 mice from each group were sacrificed, and splenocytes were aseptically collected and restimulated with immunizing antigen. Cell proliferative response was measured by MTT based assay after 3 days of incubation. ${ }^{* * *}$ Indicates significant difference compared to PBS control; ns: non-significant difference. Significance was determined at $P<0.05$. Exact probability is demonstrated on graph. B Stimulation index at 3 weeks. The stimulation index was determined at three weeks of immunization against purified L7/L12 antigen and whole Brucella cell lysate. ${ }^{* *}$ Indicates the significant difference against the PBS control, ns: non-significant difference. C Cytokine response. Mice were immunized with PBS, vector-only strain, and Salmonella secreting Brucella L7/L12. Two weeks post-immunization, 5 mice from each group were sacrificed, and splenocytes were aseptically collected and restimulated with immunizing antigen. After 1 day of incubation, interferon-gamma and IL-4 response were evaluated at mRNA level by quantitative RT-PCR. Fold change of expression is demonstrated. ***Indicates significant difference against PBS control group. Level of significance was determined at $P<0.05$. Exact probability value is demonstrated on the graph. D Flow cytometry. Mice were immunized with PBS, vector-only strain, and Salmonella secreting Brucella L7/L12. Two weeks post-immunization, 5 mice from each group were sacrificed, and splenocytes were aseptically collected and restimulated with immunizing antigen. After 1 day of incubation, cells were labeled with CD3+. CD4+ and CD8+ specific anti mouse antibodies CD3+: PE, CD4+: PerCP Vio700, CD8+: FITC (Miltenyi Biotec, Gladbach, Germany) and analyzed in Macsquant flow cytometer instrument (Miltenyi Biotec, Gladbach, Germany). Each population was gated and quantified. ${ }^{* *}$ Indicates significant difference against PBS control group. 


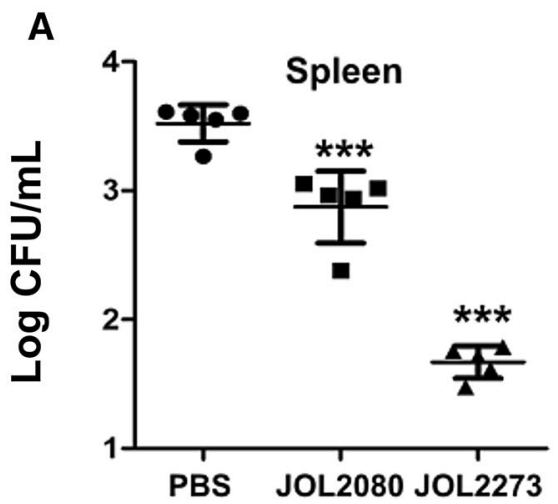

B
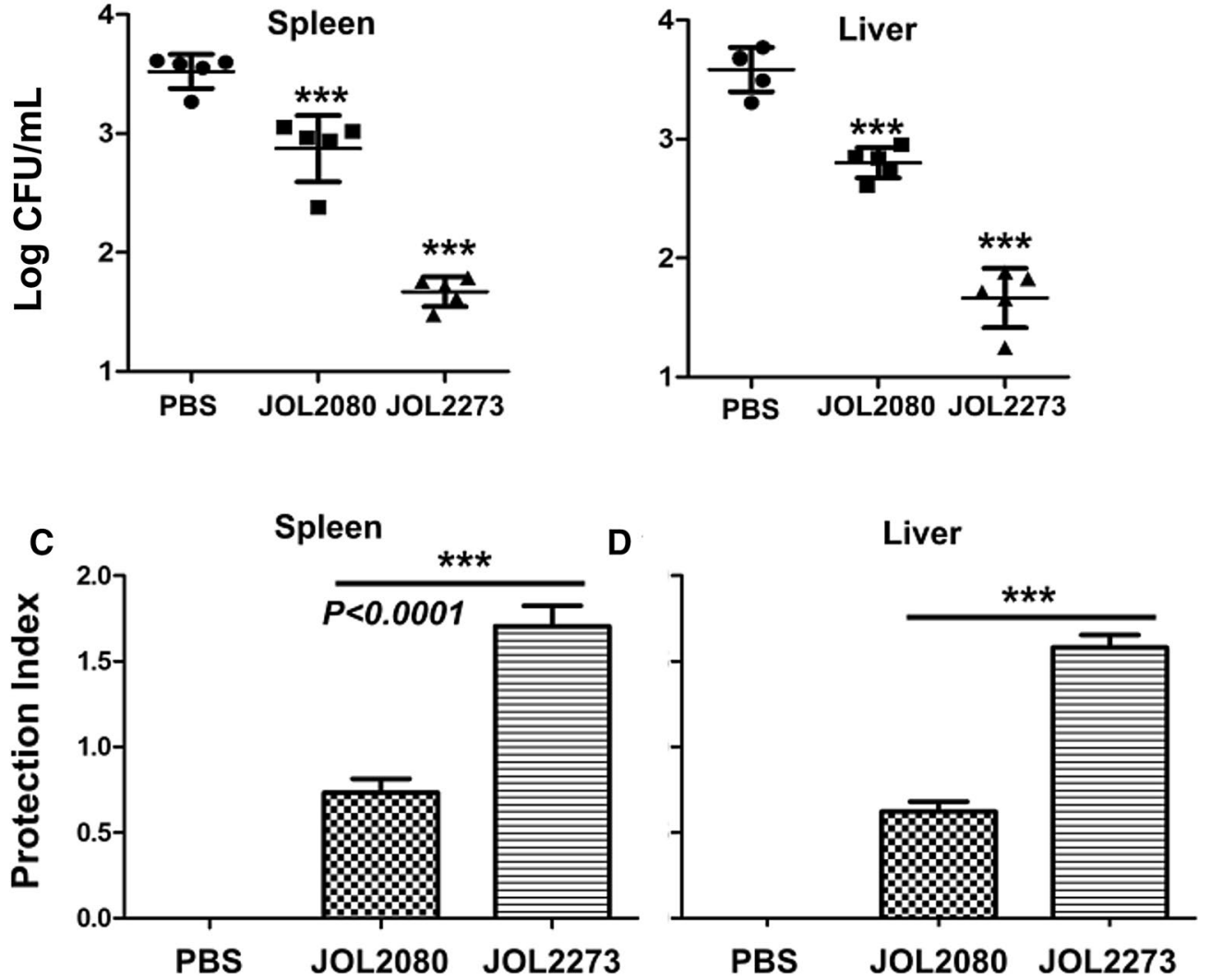

Figure 3 Protection index. Protective efficacy was determined after 2 weeks of challenge conducted on 21 st day post-immunization. Brucella colony count was taken in spleen (A) and liver (B) tissues in each group $(n=5)$. Colony counts were converted into protective index using the statistical formula $\left(y=\log (x / \log x), P I=y_{\text {pbs }}-y_{\text {test }}\right)$ and presented with standard deviation. ${ }^{* *}$ Indicates the significant difference against PBS control. $P<0.05$ was considered significantly different.

poor and insufficient for single-dose immunization [24]. This is true for both living and non-living DNA carriers during immunization procedures. Immunizations in the form of purified proteins have their limitations, such as high processing cost, requirement for multiple booster applications, and potential for degradation [25]. Such practical inefficacies were minimized in the present Salmonella mediated immunization strategy because the antigen is readily secreted by Salmonella and is available for immune stimulation (Additional file 1B). IFN- $\gamma$ mediated cellular immune responses are considered essential factors for efficient elimination of intracellular Brucella [5, 26]. This is the main objective expected from Brucella ribosomal protein L7/L12 as it can induce essential CMI responses for intracellular elimination of Brucella $[7,11]$. In the present study, immunization with Salmonella secreting Brucella ribosomal protein in a single dose clearly induced both IgG and IgA (Figure 2) humoral antibody responses [20]. These responses play a vital role in host protection during the early phase of
Brucella infection on mucosal surfaces [27]. On mucosal tissues, complement-mediated killing effectively reduces internalization of Brucella pathogen into epithelial cells. Once Brucella becomes an intracellular pathogen, the IFN- $\gamma$ cytokine-mediated Th1 immune response is crucial [28]. In the present investigation, immunization with Salmonella secreting Brucella L7/L12 protein induced both CMI (Figures 2A and B) and IFN- $\gamma$ (Figure 2C) responses significantly more than in PBS and vector control mice. An increase in the frequency of CD4+ and CD8+ T-cell populations also provides evidence for both Th1 and Th 2 immune responses. Particularly, stimulation of CD8+ markers on T-cells can be attributed to L7/L12 induced Th1 immunity, as they were significantly higher in L7/L12 immunized mice than in the vector control group. The challenge conducted with virulent $B$. abortus 544 strains showed clear reduction in Brucella populations in spleen and liver tissues in the Salmonella vaccine immunized group compared to the vector and PBS control groups, resulting in a significantly higher protection 
index (Figures 3A and D) and demonstrating the overall effectiveness of the immunization among test groups.

Considering the antigen specific immune responses in the form of humoral, CMI, and cytokine induction, Salmonella vector alone has delivered a considerably high expression levels. We hypothesize this observation may occur particularly due to amino acid sequence and epitope conservation of L7/L12 antigen between Brucella and Salmonella species (Additional file 4). These two species not only share highly similar antigenic epitopes but also demonstrate over $57 \%$ amino acid sequence identity $(95 \%$ query cover $\times 60.50 \%$ percent identity) suggesting the possibility for antibody cross reactions generated in mice against the native Salmonella L7/L12 and Salmonella secreted Brucella L7/ L12 antigen. However, the reduction in bacterial colonization in spleen and liver tissues by Salmonella vector control may be partially attributed to Salmonella induced non-specific immune stimulation signified by increased IFN- $\gamma$ response.

Safety considerations play a crucial role before live vaccine strains can reach clinical studies. The present JOL1800 strain did not induce any mortality in immunized mice due to attenuation caused by the deletion of lon, $c p x R$, and $r f a L$ genes $[13,29]$. The safety of the strain has been extensively proven in our previous studies conducted in mice models. Adding further advantage to the vaccine strain, deletion of the $r f a L$ gene confers DIVA capability [13] due to removal of a major immunodominant component from the $\mathrm{O}$-antigen. Therefore, the vaccine may be applied with minimum effects from pre-existing lipopolysaccharides (LPS specific) anti-Salmonella immunity of the immunizing host [13].

In the present study, we demonstrated that mice immunized with Salmonella secreting Brucella ribosomal protein L7/L12 could harness both humoral and CMI responses essential for the effective elimination of intracellular pathogen Brucella. Here, a single dose of immunization provides a practically convenient vaccination strategy suitable for field conditions. Further research can investigate Salmonella secreting L7/L12 fusion proteins with other immunodominant Brucella antigens, such as SodC and Omp19 [9], which may further augment L7/L12 mediated protective immunity.

\section{Supplementary information}

Supplementary information accompanies this paper at https://doi. org/10.1186/s13567-020-0735-y.

Additional file 1. Graphic representation of plasmid construct and confirmation of protein secretion. (A) Major elements of plasmid pJHL65:: L7/L12 are depicted. Figure is not drawn with actual proportions.
(B) Western blot confirmation of protein secretion. Expected size of $L 7 / L 12$ protein is $12.5 \mathrm{kDa}$.

Additional file 2. Epitope analysis of $L 7 / L 12$ is demonstrated. (A) Graphic representation of predicted epitopes is presented in yellow. (B) Distribution of predicted epitopes on three-dimensional model is demonstrated. (C) Amino acid residues resembled in each epitope are demonstrated in red. Altogether, 5 immunodominant epitopes were predicted.

Additional file 3 . High degree of $L 7 / L 12$ sequence conservation is demonstrated within Brucella taxid. Only variable residues were demonstrated in white boxes. Phylogenetic tree constructed on maximum likelihood method also demonstrates extremely high level of sequence identity. Branch length resembles the number of amino acid residue substitutions in each species. Figures were generated by Phylogeny.fr program and Unipro UGENE.

Additional file 4. Sequence homology. (A) The homology of B cell epitopes were compared between Brucella and Salmonella L7/L12 antigen. (B) The sequence identity was determined between Brucella and Salmonella L7/L12 antigen using Muscle algorithm in Ugene software. The squares depict the predicted B-cell epitopes on Brucella L7/L12 antigen.

\section{Acknowledgements}

This research was supported by Basic Science Research Program through the National Research Foundation of Korea (NRF) funded by the Ministry of Education (2019R1A6A1A03033084).

\section{Authors' contributions}

AS and JHL participated in the designing of the study. AS and $\mathrm{CH}$ performed the experiments. AS and $\mathrm{CH}$ analyzed the data. AS wrote the manuscript. AS and $\mathrm{JHL}$ reviewed and prepared the final version of the manuscript. All authors read and approved the final manuscript.

\section{Competing interests}

The authors declare that they have no competing interests.

Received: 18 September 2019 Accepted: 6 January 2020

Published online: 23 January 2020

\section{References}

1. He Y, Reichow S, Ramamoorthy S, Ding X, Lathigra R, Craig JC, Sobral BWS, Schurig GG, Sriranganathan N, Boyle SM (2006) Brucella melitensis triggers time-dependent modulation of apoptosis and down-regulation of mitochondrion-associated gene expression in mouse macrophages. Infect Immun 74:5035-5046

2. Detilleux PG, Deyoe BL, Cheville NF (1990) Penetration and intracellular growth of Brucella abortus in nonphagocytic cells in vitro. Infect Immun 58:2320-2328

3. Yang X, Skyberg JA, Cao L, Clapp B, Thornburg T, Pascual DW (2013) Progress in Brucella vaccine development. Front Biol 8:60-77

4. Murphy EA, Sathiyaseelan J, Parent MA, Zou B, Baldwin CL (2001) Interferon- $\gamma$ is crucial for surviving a Brucella abortus infection in both resistant C57BL/6 and susceptible BALB/c mice. Immunology 103:511-518

5. Prusty BR, Tabassum R, Chaudhuri P, Mohini S, Chaturvedi VK, Mishra BP, Gupta PK (2017) Expression of Omp16 and L7/L12 Brucella abortus protective antigens as secretory fusion proteins in mammalian cells. Indian J Biotechnol 16:289-295

6. Pakzad I, Rezaee A, Rasaee MJ, Tabbaraee B, Delpisheh A (2009) Immunogencity of HSA-L7/L12 (Brucella abortus ribosomal protein) in an animal model. Iran J Immunol 6:12-21

7. Lalsiamthara J, Lee JH (2017) Brucella lipopolysaccharide reinforced Salmonella delivering Brucella immunogens protects mice against virulent challenge. Vet Microbiol 205:84-91

8. Clapp B, Skyberg JA, Yang X, Thornburg T, Walters N, Pascual DW (2011) Protective live oral brucellosis vaccines stimulate Th1 and Th17 cell responses. Infect Immun 79:4165-4174 
9. Senevirathne A, Hewawaduge C, Lee JH (2019) Attenuated Salmonella secreting Brucella protective antigens confer dual-faceted protection against brucellosis and salmonellosis in a mouse model. Vet Immunol Immunopathol 209:31-36

10. Roland KL, Brenneman KE (2013) Salmonella as a vaccine delivery vehicle. Expert Rev Vaccines 12:1033-1045

11. Khan KH (2013) DNA vaccines: roles against diseases. Germs 3:26-35

12. Hur J, Lee JH (2011) Enhancement of immune responses by an attenuated Salmonella enterica serovar Typhimurium strain secreting an Escherichia coli heat-labile enterotoxin B subunit protein as an adjuvant for a live Salmonella vaccine candidate. Clin Vaccine Immunol 18:203-209

13. Lalsiamthara J, Kim JH, Lee JH (2018) Engineering of a rough auxotrophic mutant Salmonella Typhimurium for effective delivery. Oncotarget 9:25441-25457

14. Parasuraman S, Raveendran R, Kesavan R (2010) Blood sample collection in small laboratory animals. J Pharmacol Pharmacother 1:87-93

15. Tuck MK, Chan DW, Chia D, Godwin AK, Grizzle WE, Krueger KE, Rom W, Sanda M, Sorbara L, Stass S, Wang W, Brenner DE (2009) Standard operating procedures for serum and plasma collection: early detection research network consensus statement standard operating procedure integration working group. J Proteome Res 8:113-117

16. Won G, Hajam IA, Lee JH (2017) Improved lysis efficiency and immunogenicity of Salmonella ghosts mediated by co-expression of $\lambda$ phage holin-endolysin and $\psi \times 174$ gene E. Sci Rep 7:45139

17. Denizot F, Lang R (1986) Rapid colorimetric assay for cell growth and survival. Modifications to the tetrazolium dye procedure giving improved sensitivity and reliability. J Immunol Methods 89:271-277

18. Hajam IA, Lee JH (2017) Preexisting Salmonella-specific immunity interferes with the subsequent development of immune responses against the Salmonella strains delivering H9N2 hemagglutinin. Vet Microbiol 205:117-123

19. Pfaff MW (2001) A new mathematical model for relative quantification in real-time RT-PCR. Nucleic Acids Res 29:e45

20. Vitry M-A, Hanot Mambres D, De Trez C, Akira S, Ryffel B, Letesson JJ, Muraille E (2014) Humoral immunity and CD4+ Th1 cells are both necessary for a fully protective immune response upon secondary infection with Brucella melitensis. J Immunol 192:3740-3752
21. Korotkov KV, Sandkvist M, Hol WGJ (2012) The type II secretion system: biogenesis, molecular architecture and mechanism. Nat Rev Microbiol 10:336-351

22. Kaiser P, Slack E, Grant AJ, Hardt WD, Regoes RR (2013) Lymph node colonization dynamics after oral Salmonella Typhimurium infection in mice. PLoS Pathog 9:e1003532

23. Pham OH, McSorley SJ (2015) Protective host immune responses to Salmonella infection. Future Microbiol 10:101-110

24. Saade F, Petrovsky N (2012) Technologies for enhanced efficacy of DNA vaccines. Expert Rev Vaccines 11:189-209

25. Donaldson B, Al-barwani F, Young V, Hook S (2015) Subunit vaccine delivery, Chapter 7. Springer, New York

26. Ribeiro LA, Azevedo V, Le Loir Y, Olivera SC, Dieye Y, Piard JC, Gruss A, Langella P (2002) Production and targeting of the Brucella abortus antigen L7/L12 in Lactococcus lactis: a first step towards food-grade live vaccines against brucellosis. Appl Environ Microbiol 68:910-916

27. Pasquevich KA, Ibañez AE, Coria LM, Garcia SC, Estein SM, Zwerdling A, Barrionuevo P, Oliveira FS, Seither C, Warzecha H, Oliveira SC, Giambartolomei GH, Cassataro J (2011) An oral vaccine based on U-Omp19 induces protection against $B$. abortus mucosal challenge by inducing an adaptive IL-17 immune response in mice. PLoS One 6:e16203

28. Paranavitana C, Zelazowska E, Izadjoo M, Hoover D (2005) Interferon- $\gamma$ associated cytokines and chemokines produced by spleen cells from Brucella-immune mice. Cytokine 30:86-92

29. Park S, Won G, Kim J, Lee JH (2018) Potent O-antigen-deficient (rough) mutants of Salmonella Typhimurium secreting Lawsonia intracellularis antigens enhance immunogenicity and provide single-immunization protection against proliferative enteropathy and salmonellosis in a murine model. Vet Res 49:57

\section{Publisher's Note}

Springer Nature remains neutral with regard to jurisdictional claims in published maps and institutional affiliations.

Ready to submit your research? Choose BMC and benefit from

- fast, convenient online submission

- thorough peer review by experienced researchers in your field

- rapid publication on acceptance

- support for research data, including large and complex data types

- gold Open Access which fosters wider collaboration and increased citations

- maximum visibility for your research: over $100 \mathrm{M}$ website views per year

At BMC, research is always in progress.

Learn more biomedcentral.com/submissions 